\title{
Apnea and unconsciousness after accidental subdural placement of an epidural catheter
}

\author{
Seokyung Shin ${ }^{1,2}$, Youn Yi Cho ${ }^{3}$, Sang Jun Park ${ }^{1}$, and Bon-Nyeo Koo ${ }^{1,2}$ \\ ${ }^{1}$ Department of Anesthesiology and Pain Medicine, ${ }^{2}$ Anesthesia and Pain Research Institute, Yonsei University College of Medicine, \\ Seoul, ${ }^{3}$ Department of Anesthesiology and Pain Medicine, Gachon Medical School, Gil Medical Center, Incheon, Korea
}

Subdural placement of an epidural catheter is a rare complication that may lead to life-threatening consequences. However, it is difficult to detect due to the variability of symptoms and signs and insufficient diagnostic guidelines. We recently experienced a case of subdural catheter placement that caused delayed emergence with respiratory depression and mental status change.

A 56-year-old, $167 \mathrm{~cm}, 68 \mathrm{~kg}$ male patient with stomach cancer was scheduled for subtotal gastrectomy with gastrojejunostomy. The patient was recently diagnosed with hypertension and otherwise healthy. Before induction of general anesthesia, a 20-gauge multiorifice epidural catheter was inserted for postoperative analgesia through an 18-gauge Tuohy needle at the T8-9 interspace using the paramedian approach in the sitting position. The epidural space was identified using the loss of resistance technique with saline and the catheter was advanced $6 \mathrm{~cm}$ upward. After aspiration for cerebrospinal fluid (CSF) and a bolus injection of a test dose for confirmation that the catheter was not within the subarachnoid or intravenous space, general anesthesia was induced. Before skin incision, a $10 \mathrm{ml}$ epidural bolus of $1.5 \%$ lidocaine was administered and $0.15 \%$ ropivacaine with $3.6 \mathrm{ug} / \mathrm{ml}$ of fentanyl infusion was started at a rate of $4 \mathrm{ml} / \mathrm{hr}$ (Automed, ${ }^{\circledR}$ Ace Medical Co., Goyang, Korea). A total dose of $15.3 \mathrm{ml}$ of $0.15 \%$ ropivacaine and $55 \mu \mathrm{g}$ of fentanyl had been epidurally administered throughout the uneventful $230 \mathrm{~min}$ operation and an additional epidural bolus of $10 \mathrm{ml}$ of $0.225 \%$ ropivacaine mixed with $50 \mu \mathrm{g}$ of fentanyl was given at the end of the operation. The patient was extubated after he could respond to verbal commands and return of neuromuscular function was confirmed. The patient showed normal vital signs. However, several minutes after tracheal extubation, the patient developed hypertension, tachycardia and respiratory depression while he became drowsy and gradually unresponsive to verbal commands and painful stimuli. The patient showed no improvement even after 30 minutes of assisted face mask ventilation with $100 \%$ oxygen, and continued to present with unconsciousness, irregular shallow respiration and constricted pupils. Assuming that this may have been caused by the subdural spread of fentanyl, the patient-controlled analgesic device was stopped and naloxone was given intravenously in increments of $200 \mu \mathrm{g}$ at $2 \mathrm{~min}$ intervals. After $10 \mathrm{~min}$, the patient regained consciousness and adequate spontaneous breathing, and 10 min later, he was fully awake and complained of abdominal pain. After transfer to the recovery room, naloxone was continuously infused intravenously and thoracolumbar radiography was performed to identify the location of the epidural catheter with $5 \mathrm{ml}$ of radiocontrast dye (Omnipaque ${ }^{\circledR}$ ) injected through the catheter. X-ray in the lateral view showed a definite radiopaque feature in the posterior column in the subdural space (Fig. 1) and the catheter was removed. The patient was discharged on postoperative day 9 with full recovery.

The incidence of subdural block is estimated to be $0.1 \%$ after intended epidural block [1] while some report a higher incidence of $7 \%$ [2]. Characteristic presentations are reported to be a negative aspiration test, limited or marked motor block,

Corresponding author: Bon-Nyeo Koo, M.D., Ph.D., Department of Anesthesiology and Pain Medicine, and Anesthesia and Pain Research Institute, Yonsei University College of Medicine, 50, Yonsei-ro, Seodaemun-gu, Seoul 120-752, Korea. Tel: 82-2-2228-2420, Fax: 82-2-312-7185, E-mail: koobn@yuhs.ac

(c) This is an open-access article distributed under the terms of the Creative Commons Attribution Non-Commercial License (http:// creativecommons.org/licenses/by-nc/3.0/), which permits unrestricted non-commercial use, distribution, and reproduction in any medium, provided the original work is properly cited. 


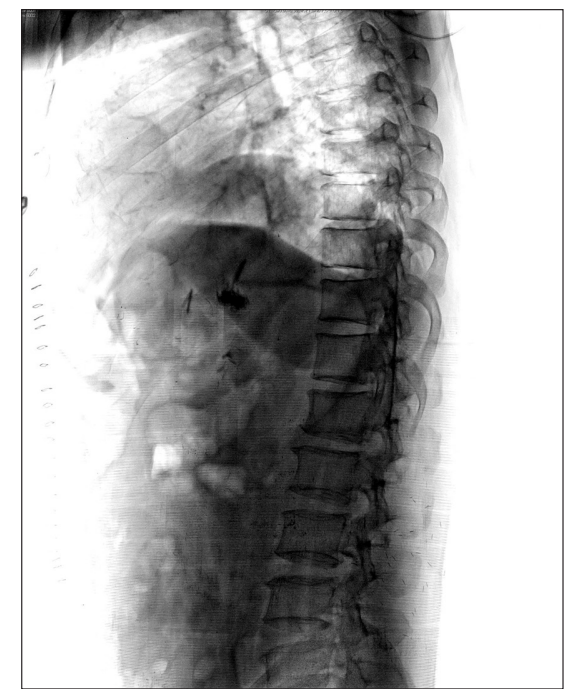

Fig. 1. Lateral view of thoracolumbar X-ray taken after radiocontrast dye injection. A thin posterior column of dye is seen within the subdural space.

moderate to severe hypotension, delayed or extreme rapid onset, progressive respiratory depression and incoordination, unconsciousness, papillary dilation, and rapid recovery of neurologic function $[3,4]$. Therefore, the clinical features vary greatly and are difficult to anticipate. Diagnostic guidelines proposed by Lubenow et al. [1] are intended for patients that are not under general anesthesia, and was therefore impossible to apply in this case. However, the radiographic findings of subdural block are archetypal with anterior-posterior views showing narrow lateral columns of dye resembling railroad tracks, usually in the thoracic and upper lumbar spine, while lateral views display thin films of dye along the dorsal and/ or ventral part of the spinal canal [3]. Although the present case only partially presents with the above-mentioned clinical features, the radiographic findings as seen in Figure 1 supported our suspicion. In addition, other possible causes of delayed awakening from anesthesia such as residual effects of anesthetics, incomplete neuromuscular relaxation reversal or hypothermia were ruled out. The discrepancy in clinical signs such as high blood pressure and constricted pupils of this case may be due to the effect of fentanyl rather than ropivacaine. The development of symptoms might have been subtle and further delayed had we not injected the bolus dose at the end of the surgery, which seems to have triggered the exaggerated spread of drug within the subdural space. Moreover, the rather low concentration of local anesthetics ( $0.15 \%$ ropivacaine) may have resulted in the weak effect of local anesthetic. However, the infusion rate and bolus dose of epidural fentanyl that was used in this case is reported to be safe from the risk of respiratory depression [5]. Although the dose of subdural fentanyl that will cause respiratory depression and mental status change is not clear, the clinical and radiographic findings of the present case and the fact that the patient was stabilized after naloxone administration strongly supports the possibility of the effect of subdurally injected fentanyl.

We suggest that subdural complications from local anesthetics and opioids should be considered when unexpected clinical features develop, and radiologic identification of the epidural catheter may be helpful when other clinical symptoms are difficult to assess.

\section{References}

1. Lubenow T, Keh-Wong E, Kristof K, Ivankovich O, Ivankovich AD. Inadvertent subdural injection: a complication of an epidural block. Anesth Analg 1988; 67: 175-9.

2. Mehta M, Salmon N. Extradural block. Confirmation of the injection site by X-ray monitoring. Anaesthesia 1985; 40: 1009-12.

3. Collier CB. Accidental subdural injection during attempted lumbar epidural block may present as a failed or inadequate block: radiographic evidence. Reg Anesth Pain Med 2004; 29: 45-51.

4. Parke TJ. Variable presentation of subdural block. Anaesthesia 1995; 50: 177.

5. Weightman WM. Respiratory arrest during epidural infusion of bupivacaine and fentanyl. Anaesth Intensive Care 1991; 19: 282-4. 